\title{
Facebook's Compatibility, Reasons for Disclosure, and Discussion of Social and Political Issues: The Case of University Students Using Facebook
}

\author{
Shuaa Aljasir ${ }^{1}$, Ayman Bajnaid ${ }^{1}$, Tariq Elyas ${ }^{2} \&$ Mustafa Alnawasrah $^{3}$ \\ ${ }^{1}$ Faculty of Media and Communication, Communication Department, King Abdulaziz University, Jeddah, Saudi \\ Arabia \\ ${ }^{2}$ European Languages Department, King Abdulaziz University, Jeddah, Saudi Arabia \\ ${ }^{3}$ Faculty of Business \& Finance, Business Administration Department, The World Islamic Science \& Education \\ University, Amman, Jordan \\ Correspondence: Shuaa Aljasir, Faculty of Media and Communication, Communication Department, King Abdulaziz \\ University, Jeddah, Saudi Arabia. E-mail: shaljasir@kau.edu.sa
}

Received: October 27, 2017

Accepted: November 8, 2017

Online Published: November 16, 2017

doi:10.5430/jms.v8n5p1

URL: https://doi.org/10.5430/jms.v8n5p1

\begin{abstract}
This paper presents the results and discussion of qualitative interviews that were conducted with 20 of Saudi university students, including findings concerning their opinions about the compatibility of Facebook with Saudi culture. It also examines their reasoning and privacy concerns when disclosing personal information online, and reports their perceptions about discussing social and political issues on Facebook. The results reveal that Saudi university students have a positive attitude toward Facebook, regard it as an integral part of their daily lives, and do not consider deactivating or deleting their accounts. Despite acknowledging the potential costs and negative consequences of sharing personal information, the majority of the respondents did not conceal their personal information on their Facebook accounts, as long as the disclosure of such information did not come to cost them too much in comparison to what they were getting from its platform. Focusing on social and political issues, the issues they discussed covered a wide range of topics.
\end{abstract}

Keywords: compatibility of facebook, reasons for disclosure, social and political issues, saudi university students

\section{Introduction}

Phenomenological interviews are among the most common strategies for collecting qualitative data. According to Creswell (1998), phenomenology is defined as individuals' understandings of the meaning of a phenomenon based on their lived experiences. Sorrell and Redmond (1995) indicate that the purpose of the phenomenological interview is to reveal common meanings among the respondents based on their lived experiences. It enables the researcher to gain a better understanding of the 'inside-out experience' of the respondents. The current research utilises this method to obtain a wealth of details regarding Saudi university students' reasons for using Facebook. As the phenomenon of Facebook usage among Saudis is still relatively unexplored, this qualitative method helps to provide a thick description and close analysis of the respondents' experiences to form a clear understanding of how meaning is generated through their embodied perceptions (Starks \& Trinidad, 2007).

According to DiCicco-Bloom and Crabtree (2006), contemporary scholars have categorised interviews into three different types: unstructured, semi-structured, and structured. This research utilises the semi-structured interview form. The semi-structured interview is defined as a qualitative form of interview in which the interviewees are asked a number of pre-set but open-ended questions (Ayres, 2008). Thus, semi-structured interviews consist of predetermined questions that are asked in a constant and systematic way, interpolated with prompts in order to stimulate rich answers by directing the interview towards the issues and themes the interviewer aims to explore.

A major advantage of the phenomenological semi-structured interview method is that it allows the interviewee to clearly understand and communicate with the interviewer, overcoming ambiguities during the interview (Wojnar \& Swanson, 2007). Besides the flexibility in collecting the data and the depth of data collected, a phenomenological 
semi-structured interview also enables the researcher to gain previously unanticipated data and investigate the research phenomenon from the perceptions of the research population (Silvermann, 1993).

A major limitation of the interview method is that it is time-consuming (Tesch, 1990). Other limitations of this method are related to the subjectivity and bias of the interviewer when interpreting the interview data. In addition, the quality of the data gathered depends on the interviewer's skills. Acknowledging the potential bias of this qualitative method, the research recorded the interviews, conducted a verbatim transcription of the data and followed Creswell's (1998) description of the systematic coding data process of phenomenological research to minimise such bias in interpreting its data. Further, the analysis was independently reviewed in order to verify the accuracy of the analysis. Conducting a pilot study also assisted in improving the quality of the data gathered by enabling the researcher and her colleagues to develop the most effective way of conducting the interviews and identifying potential problems in advance (De Silva, 2010).

\subsection{Interview Questions}

The goal of this research is to gain a detailed understanding of Saudi university students' reasons for using Facebook and to explore further any issues raised in the interview. Thus, the interviews covered three high-level topic categories based on the research objectives.

The first set of questions was related to the students' perceptions of Facebook's compatibility with their culture. They were asked about whether they had considered deleting or deactivating their Facebook accounts, what they thought about the possibility of developing a Saudi social media platform, whether Facebook is compatible with Saudi culture, and what they perceive to be the positive and negative aspects of Facebook. To elicit rich answers from the participants regarding these aspects, they were provided with a set of 118 cards with $60 \%$ positive and $40 \%$ negative or neutral words to choose from. These cards were adopted from the Microsoft reaction toolkit developed by Benedek and Miner (2002), as it consists of a wide range of possible positive and negative aspects of a social media platform. According to Barnum and Palmer (2010), previous literature on the use of the toolkit has revealed that the cards prompt users to state a rich and revealing story about their experiences. The kit also provides opportunities for discussion of the choices based on their experiences. However, it should be noted that these cards were not used in this research to measure Facebook's desirability, as this was not within the scope of the research. The cards were only used to provide further data about the potential rewards and perceived costs of using Facebook. Each participant was asked to look over the cards that were spread out in a random pattern, select up to five cards based on what they liked or disliked about Facebook, and explain what each card meant to them.

The findings from a previous research had revealed that the students tended to disclose a high amount of their personal information on Facebook (Aljasir et al., 2017a). Therefore, the second set of questions focuses on understanding more about their reasons for such disclosure and whether they had privacy concerns.

The results of another pervious research had indicated that discussing political and social issues were important gratifications for the students (Aljasir et al., 2017b). Thus, this research seeks to understand the role of Facebook in enabling more open discussions and other users' reactions to such usage.

\subsection{Pilot Study of the Interviews}

A pilot study was conducted with ten volunteer students (five males and five females) aged between 18 and 22 (Mean $=19.80$ years, $\mathrm{SD}=1.64$ ) for several reasons. It helped in identifying any problematic questions that were ambiguous or that made participants uncomfortable. For example, during the pilot interviews, the researcher found that it was necessary to explain and clarify the interview question: what do you think about developing a Saudi social media platform? because it was unclear to the participants whether this question regarded the establishment of a Saudi social media platform as an alternative to Facebook or in addition to Facebook. The question was reworded as: do you think there is a need to develop a Saudi social media platform instead of using Facebook? The pilot study also assisted in forming operational procedures and solving emergent problems prior to conducting the main study. For example, from the pilot study it became clear that some interview questions needed more prompts to encourage dialogue.

To ensure the reliability of the cards, a retest of the cards was conducted with the same ten students two weeks after conducting the main pilot study. The measure of agreement kappa was used as a statistical method to evaluate the test-retest reliability. The kappa reliability coefficients ranged from 0.81 to 1.00 , with most cards having perfect reliability (1.00). 


\subsection{Administration of the Interviews}

The students were asked to volunteer to participate in audio-taped interviews regarding their perceptions about the reasons for using Facebook. Participant information sheets and consent forms were sent via email to those who showed interest in participating. A total of 20 volunteers ( 10 males and 10 females), who were aged between 19 and 23 (Mean $=20.85$ years, $\mathrm{SD}=1.35$ ), took part during the autumn semester of 2013-2014. According to Green and Thorogood (2009), interviewing 20 participants is considered a sufficient number among qualitative researchers. In particular, for phenomenological interview studies, Starks and Trinidad (2007) indicate that typical sample sizes range from 1 to 10 respondents. Thus, the sample size of the current phase was considered to be adequate.

A suitable time and place was arranged for conducting the interviews. Locations were chosen that were convenient for the participants and typically took place in a private area on campus. All the interviews were recorded. Before the beginning of each interview, the researcher and her colleagues reviewed the purpose of the interview, provided an overview of the issues that would be covered and reminded the interviewees that they had the right to not answer any of the interview questions if they felt uncomfortable or if they chose not to disclose specific information. All of these points were also stated in the participant consent form, which the participants were asked to submit. The duration of the interviews was between 40 and 50 minutes, followed by a debriefing session.

\subsection{Analysis of Interview Transcripts}

After all the interviews were conducted, a verbatim transcription was completed and copies of these transcripts were sent to the participants to read and confirm that they accurately reflect their responses. Following Creswell's (1998) description of the systematic coding data process of phenomenological research, the responses were grouped together and coded to look for patterns in the data. Codes, categories, and themes emerged through the inductive analysis of this data, allowing the researcher to capture the essence of the subject's experiences with Facebook. The transcripts were then read twice to search for any further codes, categories, or themes. Rich description was used, involving reporting details of the interviews through the inclusion of quotations from the participants. The report was independently reviewed to verify the accuracy of the codes, categories, and themes suggested by the researcher's findings.

The following sections present Saudi university students' perceptions regarding the compatibility of Facebook with Saudi culture, their reasons for disclosing personal information on Facebook, and their perceptions of discussing social and political issues on Facebook. The research ends with the conclusion of this research.

\section{Compatibility of Facebook with Saudi Culture}

According to Chiang (2013) and Alalwan et al (2016), public perceptions of a given social media platform have the strongest direct effect on users' intention to continue utilising that platform. The current section presents the results of the data analyses and a discussion of interviewees' responses regarding whether they have considered deleting or deactivating their Facebook accounts, whether they would prefer to use a Saudi social media platform rather than Facebook, and whether using Facebook contradicts Saudi culture. The respondents were also interviewed about the positive and negative aspects of Facebook to further examine how they perceive and evaluate its potential rewards and costs.

\subsection{Consideration of Deactivating/Deleting Facebook Accounts}

According to Facebook's privacy policy, when users want to stop using their accounts, they can either deactivate or delete them. When a user deactivates a Facebook account, other users will not be able to view it, but all of its information will be saved in the Facebook database in case the user decides to reactivate the account. When a user chooses to delete the account, in contrast, it is permanently deleted from Facebook, and there is no way to reactivate it (Facebook, 2013). This section assists in both examining whether the rewards Saudi university students obtain from using Facebook keep them from deactivating or deleting their accounts and determining what rewards they would miss most if they left the platform. When the current respondents were interviewed about this issue, they all indicated that they would not delete their Facebook accounts, and they also had not deactivated them. They provided two main reasons for maintaining their Facebook accounts: first, they regard it as an effective way to communicate and be updated about others' lives. Second, they perceive the rewards gained from using this social media platform as outweighing its disadvantages.

With regard to the first reason, the participants explained that Facebook gives them access to a mass audience without the need for individual contact information. This is true regardless of whether their contacts consist of people drawn from their offline networks or users they have only met online and friended. Thus, they consider reaching and having access to a wide audience a valuable reward that will assure their continued use of Facebook. For example, 
one of the participants stated, "Facebook is the main way I have to communicate with a considerable number of my friends" (F02). The participants also indicated that Facebook is a very effective tool through which they can inform their social networks about their daily practices. They indicated that deleting their accounts would be counter to this desired gratification. They would also lose the advantage of receiving information about their friends. As one of the interviewees stated, "How can I know others' updates . . . if I deactivate it?" (M07). This reward is an example of what Ellison, Steinfield, \& Lampe (2007) call 'maintained bonding social capital', which is an individual's capability to continue contacting members from their offline world.

Facebook represents a significant shift in communication from verbal face-to-face communications to digital one-to-many communications, and the respondents regarded this as a significant advantage that made them keen to maintain their accounts. Such a one-to-many form of communication offers them an effective venue for establishing and maintaining numerous social contacts (Winter, et al. 2014; Obeidat, Tarhini, \& Aqqad, 2017). In Chiou \& Lee's (2013) investigation of the prevalence of one-to-many communication on Facebook among 102 undergraduate Taiwanese Facebook users, 92.8\% of the users reported that their most frequent method of content distribution was sharing information with their friends. As Facebook combines the features of both mass and personal media, the convergence has resulted in blurred boundaries between one-to-one and one-to-many communication (Jenkins, 2006). For example, Facebook's status update function is a tool to broadcast a personal message from one to many. Thus, the possibility of reaching all of one's Facebook friends with a single status update has similarities to traditional broadcasting and is different from personal one-to-one communication tools such as the written letter and the telephone. Facebook users differ from a broadcast audience, however, in the sense that they combine the roles of both the producer and audience of content. They are identified as friends, not audiences, and have their own profiles and online presence. This finding confirms the results obtained from previous research, which reveals that communicating with others; sharing personal updates, and investigating other users are among the most common gratifications obtained from using Facebook.

With regard to the second reason for the continued use of Facebook, users indicated that the advantages of using Facebook in their personal, social, and academic lives are much greater than its disadvantages. Such a principle, in which rewards and costs are determined in order to decide whether to continue using Facebook, is in line with the assumptions of social penetration theory. According to this theory, unless the individuals find the activities they engage in to be profitable, they will not continue to engage in them. The individual who experiences a 'loss' (i.e. finds an activity more costly than it is rewarding) will have an incentive to withdraw from the interaction (Altman \& Taylor, 1973). Although the respondents felt that using Facebook leads them to disclose a considerable amount of their personal information because it encourages them to engage in multiple interesting activities that require revealing several aspects of their identities, they perceived themselves as capable of coping with the potential costs associated with disclosing their personal information. This finding is also consistent with the results of a study conducted by Shi, Xu, \& Wang (2013), in which the authors conducted content analysis on 308 Facebook comments by American users related to their privacy concerns and noted that, although their participants actively engaged in Facebook, as soon as they felt that their usage was going to have more negative consequences, they would use it more sparingly, deactivate their accounts, or even delete them.

From these findings, it can be seen that Facebook is highly valued by the participants and forms an integral part of their daily practices. This finding is consistent with Romano's (2013:6) argument that "social media is not just a temporary fad or an alternative communication tool; instead, it can now be viewed as one of the primary communication vehicles for delivering information, data, and messages to a worldwide audience at a moment's notice".

\subsection{Saudi Social Media Platform vs. Facebook}

As Saudi culture has distinct traditions and customs that shape its citizens' values and attitudes, the participants were interviewed regarding whether there is a need to create a Saudi social media platform as an alternative to Facebook, as has happened in some other countries, such as Turkey, China, Russia and Malaysia. The majority of the participants did not support this idea and justified their position in two ways: first, they believed that Saudis should not cut themselves off from other cultures. Second, they did not believe that such a platform would be as advanced and popular as the global Facebook platform.

With regard to online cross-cultural communication, the interviewees perceived that relying on a Saudi social media platform for communication (rather than an international platform like Facebook) would isolate them, deny them the privilege of interacting with people from different cultures, learn about other cultures, and prevent them from presenting their Islamic culture and its values in a positive fashion. The respondents used phrases like "isolate 
ourselves from the rest of the world" (F01) and "will then be a closed society" (F10). It is interesting that the findings show that these young and educated people actively strive for the cross-cultural interaction that is possible in an international virtual space. The majority perceive the idea of a national platform as a step backward from an open, international form of communication to a closed and limited one. They rejected such an idea, especially after having experienced communication with users from different cultures. In fact, the majority of respondents view cross-cultural communication as a further reason for using Facebook.

According to Enli \& Thumim (2012), a key feature of Facebook is the mixture of local, regional, national, and global content, and typical users often express themselves in a mixture of their mother tongue and international languages (mostly English) when interacting on Facebook. Besides, Facebook is available in over 40 different languages and is profoundly global in its structure, unlike national media which tend to be monolingual and culturally specific. Thus, Facebook users may have contacts in their 'friends list' from outside the traditional social and national borders of their home environment. Although the reality of online social worlds varies - some users of Facebook, for instance, develop more international networks and ties than others - the global reach of Facebook seems to be highly emphasised in conservative cultures such as Saudi society. Facebook and other social media platforms could be considered their window to the world, and at the same time, a window for the international world to the events in these societies. This advantage offered by international social media platforms, such as Facebook, Twitter, and YouTube, appears to make them preferable over similar, existing national social media platforms, as was seen in recent events in Turkey. It was better for Turkish citizens to inform international organisations of what was going on in Turkey through these relatively uncensored platforms than to share news through their national social media platform (Guillet, 2013; Al-Badi et al., 2017).

The second reason why the respondents did not support the idea of a Saudi social media platform is because they feel that it would be less technologically advanced. The respondents perceived that a Saudi-specific social media platform would require a lot of time before it was advanced and attractive enough to compete with Facebook. It would need to include new and more novel features to attract a large number of users. That Saudi university students prefer to use Facebook over a national platform is consistent with research conducted by Saw et al. (2013), which showed that, although a sample of international Chinese students had access to a China-based social media platform (Renren), they preferred to use Facebook to communicate with others, including other Chinese people. These findings are also in keeping with research on Malaysian users that indicated that a mere $10 \%$ of participants made use of the local network platform while $60 \%$ possessed accounts on Facebook. According to this study, Malaysian people were not aware of the national social media platforms, and the national platforms generally fail to get a good response from consumers (Mustafa \& Hamzah, 2011).

Some Saudi university students did perceive value in creating a Saudi social media platform. They believed that such a platform could prevent younger users from being exposed to harmful Western material. This was exemplified by the following comment: "Our generation can easily be exposed to too many harmful actions. So, it is better to create a Saudi social media platform in order to be able to control it" (M01). They believe that creating a Saudi Islamic social media platform would eliminate most of the negative consequences associated with the international social media platforms. In line with this notion, a Saudi journalist, El-Shenawi, asserted that international social media platforms such as Facebook and Twitter should be supervised by the nation's religious police and that the aim should be to get rid of harmful accounts that encourage pornography and magic. She argued that the subject matter contained in these international social media platforms is not easy to control and purify and that it will ultimately damage the youth who constantly use these social media platforms (El-Shenawi, 2014). However, such reservations have not received official support for regulating social media content to date.

\subsection{Compatibility of Facebook Usage with Saudi Culture}

Older technological innovations such as radio, television, and Web 1.0 faced rejection from a large segment of Saudi society upon their introduction, as they were perceived as a threat to the society's culture (Rathmell, 1997). In contrast, social media in general and Facebook in particular have been welcomed to the point that Saudi religious clerics have large numbers of friends on Facebook and followers on Twitter (Coleman, 2011). According to Schanzer \& Miller (2012: 46), "Despite their opposition to the morally hazardous social media, both sanctioned and unsanctioned clerics now take to the internet with zeal. Particularly, the unofficial clerics have leveraged Twitter, Facebook, YouTube, and iPhone apps to propel their global influence and reach". Since all of the respondents in this research confirmed the compatibility of Facebook use with the values and culture of Saudi Arabia, they were asked to explain why, and offered two main answers: the privacy settings and Saudis' trend towards openness nowadays.

Some of the current respondents perceived the privacy settings as the perfect tool to bring Facebook in line with 
Saudi culture. They explained that, because they could use privacy settings to hide part of their personal information in their profiles that would be inappropriate to disclose in public, their Facebook use does not conflict with their cultural values. It seems likely that some interviewees perceive the use of Facebook without any adjustments (e.g. configuring the privacy settings to withhold personal facts) as incompatible with their values and traditions. It is only when respondents use the privacy settings to properly configure their accounts that usage becomes culturally acceptable and complies with the norms and dictates of Saudi culture. Because the respondents believe that "you can customise it through privacy settings to be in line with our culture" (M10), it could be argued that an individual's use of Facebook determines its cultural compatibility. The majority of the respondents believe that Facebook is in line with the norms of Saudi society because the contemporary society is more open, modern, and willing to accept the new norms that this new media platform brings.

These two sets of responses show that users can be divided into those who wish to maintain the traditions of society by utilising the privacy settings and those who want to move towards a more open perspective. This reflects growing diversity in the wider Saudi society: scholars are inclined to divide Saudi citizens according to their attitudes toward modernity and new technology into conservatives and liberals (Yamani, 2010).

\subsection{Positive and Negative Aspects of Facebook}

To gain a further understanding of the rewards Saudi university students obtain and the potential costs they pay for Facebook usage, cards from the Microsoft toolkit were used to prompt users to state a rich and revealing story about their experiences with Facebook. The cards feature positive and negative adjectives that enable interviewees to indicate their perceptions of Facebook's positive and negative or neutral words. Each participant was asked to look over the cards, spread out in a random pattern, and select up to five of them based on what they liked or disliked about Facebook, and explain what each card meant to them. Analysing the results revealed that positive aspects could be grouped under five main headings: attractiveness, ease of use, customisability, helpfulness, and advanced technology. On the other hand, the main negative aspects related to lack of security, impersonality, uncontrollability, distractions, frustration, and annoyance. The use of these terms is discussed below, as the terms are not always used in a standard way, and as such reveal more insights in to the perception of Facebook.

The students elaborated on their perceptions of Facebook's attractiveness by stating that its perceived benefits had attracted so many Saudis to join its community that it was rare to find someone who did not have a Facebook account. Respondents indicated that Facebook can also be used to invite support for current affairs, whether social, religious, political, or educational, because users will find a large number of people to hear their voices, engage in their causes, and actively interact in debates. Thus, any topic raised on Facebook will garner the attention of a large number of people. Zuckerman (2014) described this large population of users in terms of a latent capacity. The analysis of the status updates previous research revealed that Saudi university students are indeed willing to engage in these discussions. This positive aspect of Facebook is in line with Gillan, Pickerill, \& Webster's (2008) distinction between the manifest function and latent capacity of new technology. While the manifest function of Facebook could be to socially communicate with others about causes, it is the latent capacity that facilitates changes in people's lives.

Facebook was also described as being easy to use because the steps to utilise it, from the first stages of registering and setting up an account to generating content and interacting regarding shared information, are very simple and clear. Thus, it could be argued that, regardless of their level of technical competence, Saudi university students believe they can effortlessly broadcast their news and messages. This feature was also one of the prominent positive aspects of social media illustrated by Zuckerman (2014). He indicated that, because social media platforms are designed for millions of inexperienced users, such users are able to easily use social media platforms to publish content. Gauntlett (2011: 13) argued that such "easy to use online tools which enable people to learn about, and from each other, and to collaborate and share resources, have made a real difference to what people do with, and can get from, their electronic media".

Customisability was considered to be another positive aspect of Facebook in that, through this feature, users can customise the information accessed by them and by other users. Such customisation takes two forms: first, Facebook allows users to select the audience that can view their profile and status updates. Second, the news feed can be customised to display only the content that matches their interests.

Facebook was considered to be helpful because it allowed students to organise cooperative work (such as voluntary missions and awareness campaigns) and spread information to a large number of users through the distribution of articles, sharing of status updates, and creation of informative pages to which others can subscribe. This benefit has been highlighted by Shirky (2011), one of the prominent advocates of the helpfulness of social media, who indicates that distributing information on social media platforms, even when it is done among groups with weak ties, helps in 
achieving goals that were unattainable before the existence of this technology.

Facebook was considered advanced because it frequently introduces new updates and users upload new content every day. Additionally, it is a stable platform that has rarely gone down. Such an explanation is in line with the current sample's earlier justification for asserting that creating a Saudi social media platform would require considerable time, money and effort if the platform were to be as advanced as Facebook.

When asked about its negative aspects, the students declared that lack of security was one of the most negative aspects. Users believe that their privacy could be threatened if their disclosed personal information were leaked to and misused by - third parties. Despite disclosing a considerable amount of their personal information, the respondents do not feel certain that their information was safe and believed that it might be misused. Facebook was also perceived as being impersonal. The respondents' definition of this was related to the loss of ownership of information and users' inability to prevent Facebook from giving their information to others. They acknowledged Facebook's actions in this, saying that "it is a matter of doing business rather than offering a free space for individuals to interact in" (F04). They were also aware that Facebook as a company viewed and stored their information and could sell it. Such an acknowledgment is consistent with the previous literatures revelation that being a Facebook member is not actually free of charge, because users contribute their information as the price of joining it (e.g., Doyle \& Fraser, 2012; Scholz, 2012).

Saudi university students also perceived uncontrollability to be a negative aspect of Facebook. They commented that they could not control the information they had generated and that information used to raise awareness may have been subsequently misused to criticise a cause and show its negative side, leading to virtual anti-campaigns that undermine the cause in question and make users less convinced of its agenda. This was clearly highlighted with the campaign for Saudi women's right to drive cars, in which Saudis opposed to women being allowed to drive created oppositional campaigns to undermine the campaign (Oct26driving, 2013).

This finding shows that the fifth assumption of uses and gratifications theory, which states that people are typicallybut not always - more influential than the media (Rubin, 1994), could be updated to include the impact of the content generated by different users. As social media platforms depend mainly on ordinary users to contribute their content, this content does not necessarily follow an institutional agenda or go through gatekeepers as in the traditional media. The interactive nature of social media platforms may complicate the typical impact of media content because social media users are exposed to a wide range of issues and diversity of opinions regarding these issues. Nevertheless, it should be noted that, while the corporate media bias may have been minimised in social media platforms, each user who contributes on Facebook has a position or agenda as well. This may change as the posts of others may lead to a well-rounded discussion.

Facebook was also perceived to be distracting and as such inhibited the students' ability to focus on a particular post. Continuous newsfeed updates meant that respondents were distracted from engaged reading or commenting on debates by potentially more interesting topics. This limitation has also been noted by Zuckerman (2014), who indicates that one of the biggest limitations of social media is that, while social media platforms enable users to generate and share content, they do not guarantee that this content will be viewed and capture the attention of other users.

The first assumption of uses and gratifications theory is that "communication behaviour, including the selection and use of the media, is goal-directed, purposive, and motivated" (Rubin, 1994: 428). These results suggest that, in the case of social media usage, this assumption could be developed further. While Facebook users may be goal-directed and motivated to start using Facebook, their usage may become more unplanned or less purposive as they are assaulted with distracting, unsolicited content. Hence, the interactivity and immediacy of the platform may distract them from achieving their main aims. These actions may even lead them away from Facebook to other media tools, such as online newspapers. Mitchell \& Page (2013) confirmed that about $64 \%$ of online newspaper readers are being directed to these websites from Facebook.

Saudi university students perceived Facebook as frustrating mainly due to the less developed Saudi technological infrastructure that meant that connections were lost. Respondents found this very frustrating when they were in the middle of a heated discussion. They indicated that, despite paying fees for Internet services, they cannot remain continuously online. It should be noted that this limitation is, however, more of a negative aspect of the country's infrastructure than a negative aspect inherent to the Facebook platform.

The participants also described Facebook as being annoying because they felt pressured by its notifications, updates, and messages. Even if they have the choice to switch notifications off, they still feel responsible for replying, 
commenting, liking, and responding to other Facebook users. Such actions may fulfil their Facebook friends' needs for attention and also Facebook's goal of winning the competition with other media platforms by compelling users to be on Facebook as often as possible. These findings support the fourth assumption of uses and gratifications theory that "the media compete with other forms of communication, or functional alternatives such as interpersonal interaction, for selection, attention, and use" (Rubin, 1994: 428).

Utilising the cards to reveal the positive and negative aspects of Facebook assists in investigating the applicability of uses and gratification theory's assumptions regarding the usage of a social media platform and suggests further modifications to its assumptions. It also helps in revealing the extent to which Facebook users are purposive and goal-oriented in obtaining expected gratifications. Additionally, the positive and negative aspects of Facebook perceived by the current sample offer a further insight into the key features of this social media platform, which afford Saudi university students opportunities to obtain diverse gratifications, and the perceived costs from using it that may possibly hinder them from obtaining such gratifications.

\section{Reasons for Disclosing Personal Information on Facebook and Users' Privacy Concerns}

Social media platforms have altered the way of disclosing and sharing personal information with others. Unlike emails, where personal information is mainly shared directly with the recipient of the communication, personal information in social media platforms is potentially shared with a massive audience. Users are even encouraged by the service providers to disclose and share a substantial amount of personal information with other users (Kisilevich, Ang, \& Last, 2012). In a previous research, the results revealed that Saudi university students did disclose a large amount of their personal information (Aljasir et al. 2017a). Therefore, this section addresses interviewees' explanations of their disclosure of personal information and their privacy concerns regarding such disclosures.

\subsection{Reasons for Disclosing Personal Information on Facebook}

The concept of self-disclosure has a long history in social science research. Reno and Kenny (1992) indicated that interest in this phenomenon had its roots in Lewin's (1935) cross-cultural study of the differences between Americans and Germans in their degree of openness with newcomers. Such interest in understanding self-disclosure behaviour was revived thirty years later following the publication of Jourard (1964), which emphasised the role of self-disclosure in personal relationships and in the maintenance of psychological health and personal development. In the 1970s, researchers started to be more interested in understanding the processes involved in self-disclosure. This was reflected in Cozby's (1973) classic definition of self-disclosure as any personal information that someone verbally communicates to someone else. Cozby presented self-disclosure as a process and suggested that this process impinges on both the person who reveals and the person who receives the information.

Around the same time, Altman and Taylor (1973) developed social penetration theory, following the social exchange perspective of Thibaut and Kelley (1959). Instead of focusing on the cyclical process of self-disclosure, they were interested in investigating individuals' levels of disclosed personal information and the role of costs and rewards in determining such disclosure within a definite setting. Their theory assumes that individuals are rational beings, who constantly try to maximise their own rewards and minimise their costs.

Disclosure within social penetration theory is considered as having two dimensions: breadth and depth. Breadth refers to the amount of information individuals reveal about themselves or the number of topics they disclose, while depth refers to the degree of sensitivity of the disclosed information. The theory posits that there is a linear increase in both the breadth and depth of self-disclosure when individuals expect favourable outcomes from the disclosure. The 'onion analogy' is used to explain such increase: individuals start with the outer layers, disclosing basic information about themselves, and when they experience rewards that outweigh this cost, they reveal more sensitive inner layers of their personal information (Altman \& Taylor, 1973). Based on this analogy, individuals do not reveal sensitive information about themselves and shed these layers all at once. Instead, they tend to maintain protective outer layers around a central core that signifies their inner selves until they obtain rewards that lead them to take the risk of revealing more about themselves.

Since its articulation, several attempts have been made to examine the assumptions of social penetration theory in offline one-to-one communication as well as in one-to-many interactions (e.g., Morton, 1978; Hays, 1985; Hammer \& Gudykunst, 1987; Labianca \& Brass, 2006). Nevertheless, the theory has been criticised for its notion that individuals are rational beings in their one-to-one interactions. Critics argue that individuals are not always rational in evaluating the rewards and costs they experience from disclosing personal information when it comes to intimate interactions (Strom, 2002; Kim \& Yun, 2007). As these criticisms are directed towards the applicability of social penetration theory within one-to-one interactions, it is worth investigating whether individuals tend to be more 
rational in their self-disclosure behaviour in one-to-many communicational settings.

With the development of internet technologies in general and the diffusion of social media in particular, scholars have transferred their attention to the applicability of this classic offline theory to online settings (Wang et al., 2012). Although social penetration theory was initially proposed to explain self-disclosure in offline settings, scholars have argued that it provides a suitable frame for studying self-disclosure in social media contexts. The theory has been widely used in online social media contexts to examine one-to-one interaction (e.g. Cho, 2010; McEwan, 2011; Kim et al., 2012; Chen \& Sharma, 2013; Limperos et al., 2014) and one-to-many communications (e.g., Thotho, 2010; Tang \& Wang, 2012; Jin, 2013; Olson, 2013).

While users in social media platforms are not required to disclose a certain amount of their personal information, the current research argues that the gratifications (or 'rewards' in the terminology of social penetration theory) they obtain from initial disclosure encourage them to disclose increasingly deeper layers of their personal information. Several costs have been identified as the result of disclosing such information. Gross and Acquisti (2005) identify three potential sources of costs of disclosing personal information on social media platforms: the hosting site, the user's friends, or third parties. The hosting site, in this case social media service provides, can easily access users' information and collect data about them. Members of the user's network can misuse disclosed information, which could lead to online stalking, bullying, stigmatisation, identity fraud, criminal charges, and sexual predation. Third parties, from hackers to governmental agencies, can access a user's information for their own agendas with or without the hosting site's direct agreement. This research attempts to investigate whether users are aware of the negative consequences of disclosing personal information and the extent to which they are concerned about the privacy of their disclosed information, in order to reveal the extent to which such concerns might deter them from sharing their personal information online.

In conjunction with social penetration theory, Nosko, Wood, and Molema (2010) developed a classification of disclosed personal information specific to Facebook. They divided personal information into three broad levels according to its sensitivity: basic personal identifying information, sensitive personal information, and potentially stigmatising personal information. According to Christofides, Muise, and Desmarais (2009), making the choice between the concealment and disclosure of personal information and determining the depth and breadth of disclosure is a balancing act for individuals between costs and rewards.

Several studies have investigated the self-disclosure behaviour of university students on Facebook. Some of these have attempted to investigate one or both dimensions of self-disclosure (breadth and depth). Other studies have focused on the relationships between privacy concerns and disclosure. A review of these studies is provided below.

Focusing mainly on the breadth of self-disclosure, Kolek and Saunders (2008) used quantitative content analysis to examine the disclosure behaviour of 50 identifiable information items among a random sample of American university students' Facebook profiles $(n=464)$. The results of this study revealed that they disclosed a substantial proportion of their identifiable information, including contact residence information, course schedules, positive mentions of their university, and images of students drinking alcohol. The study also showed that a leak of the disclosed information to unknown viewers could lead to positive or negative consequences but it did not investigate how participants regarded the potential impacts of such disclosure behaviour or how they would modify their behaviour in light of perceiving such consequences.

Using social penetration theory, Thotho (2010) conducted a cross-cultural content analysis of 500 Kenyans' and Americans' Facebook profiles to compare the breadth of information they disclosed online. The results revealed that users from both cultures tended to disclose their demographic variables, but that Kenyans were more likely to use a self-portrait on their profiles and disclosed more information about their religious and political views. On the other hand, a much higher percentage of Americans revealed their full date of birth and information about their college education, such as college name and years of enrolment. Users from both cultures showed low levels of disclosure of their contact information. Thotho's (2010) study highlighted the role of culture in revealing religious and political affiliations by comparing self-disclosure behaviour between a relatively conservative culture and a more liberal one. While she indicated that her study aimed to adopt the assumptions of social penetration theory, Thotho (2010) did not analyse the disclosed information according to its breadth and depth.

Day (2013) also conducted a cross-cultural study to compare how willing Facebook users are to disclose personal information among a sample from Canada, India, Portugal, Australia, New Zealand, and the United States (n = 27). Although the majority of the sample indicated that they disclosed information about their daily lives, the results showed that they did not share problems relating to personal relationships, health, work or family matters, or their religious beliefs. While this study did not classify the disclosed information according to its sensitivity, the findings 
demonstrated that users avoided disclosing information related to their problems, which may indicate that users are rational in their decisions about sharing some information and avoiding the disclosure of other information.

Olson (2013) adopted social penetration theory and utilised both questionnaire and focus group methods with American participants to investigate the reasons why users disclose personal information on Facebook and how this affects their self-esteem. Eighty-one of the participants responded to the survey and reported that they disclose their positive qualities on Facebook because this makes them feel good. The 15 focus group participants also indicated that such disclosure on Facebook had a positive effect on their self-esteem. This study confirms the assumption of social penetration theory that individuals tend to disclose more when they expect positive outcomes. Although it demonstrated the relationship between a potential reward users may obtain from Facebook and the breadth of their self-disclosure behaviour, Olson's (2013) study did not determine the depth of self-disclosure or the degree of the sensitivity of the information. Determining the relationship between the rewards and the depth of self-disclosure would provide a comprehensive picture regarding the extent to which these rewards are evaluated by users.

It is notable that the above studies mainly focused on counting the frequency of certain disclosed personal information items on Facebook and neglected others. They also did not distinguish between the levels of the depth of the disclosed information. Such limitations were addressed by Nosko, Wood, and Molema (2010), who analysed the breadth and depth of information disclosure on Facebook. Using factor analysis, they divided disclosed information items into three broad levels according to their sensitivity: basic personal identifying information, sensitive personal information, and potentially stigmatising personal information. Applying this classification to the disclosed information of 400 randomly selected Canadian Facebook profiles, Nosko, Wood, and Molema (2010) found that their sample tended to disclose $48.2 \%$ of their basic personal identifying information, $69.8 \%$ of their sensitive personal information, and $45.2 \%$ of their potentially stigmatising personal information.

Ntlatywa, Botha, and Haskins (2012) applied this classification system to their analysis of self-reported information disclosure as compared with observed information disclosure on Facebook. Their study of 131 South African university students showed that observed information disclosure was in fact $30 \%$ greater than self-reported information disclosure. This indicates that users may not accurately report the information they disclose (given that they were off by nearly a third), with the exception of their friends list, which was the only Facebook information item where the observed information disclosure scored lower than self-reported information disclosure.

Researchers interested in examining the relationship between users' privacy concerns and their level of disclosure of personal information on Facebook have mainly utilised self-report methods and conducted their research with university students. These studies have yielded conflicting results. Some have revealed that students' privacy concerns and information disclosure are negatively correlated. For instance, studies conducted with 210 German university students (Krasnova et al., 2009); with 122 American university students (Stutzman, Capra, \&Thompson, 2011); with 450 American university students (Tufekci, 2012), and with 77 Canadian university students (Young \& Quan-Haase, 2009) all indicated that students with the greatest privacy concerns disclosed the least information. Studies conducted with Islamic samples also revealed similar results. For instance, studies conducted by Osman and Ab.Rahim (2012) with 30 Malaysian university students and by Mohamed (2011) with 325 Emirati and Egyptian users revealed a negative relationship between online privacy concerns and disclosure of personal information online.

Other studies have noted a privacy paradox - a term proposed by Barnes (2006) to refer to users who claim to be concerned about their online privacy but still disclose a considerable amount of personal information on their profiles. For instance, studies conducted with 50 American university students (Govani \& Pashley, 2005), with 13 American university students (Strater \& Richter, 2007), and with 343 Canadian university students (Christofides, Muise, \& Desmarais, 2009) revealed that while participants reported awareness of some of the privacy concerns associated with Facebook, they highly disclosed personal information on their Facebook profiles.

A noticeable drawback of the studies that revealed a negative correlation between self-disclosure and privacy concerns online and the studies that demonstrated a privacy paradox among their samples is that they all depended on self-reporting methods to collect their data. According to Ntlatywa, Botha, and Haskins (2012), one of the shortcomings of studies that utilise self-reporting methods in investigating self-disclosure is that participants may not accurately recall the exact amount of their disclosed personal information, which may lead them to evaluate it in a way that does not match their actual behaviour. Such an outcome has been clearly shown in Ntlatywa, Botha, and Haskins's (2012) study, which reveals a significant difference between self-reported data regarding disclosed information on Facebook and the data collected from observing the participants' actual Facebook profiles.

According to Al-Khateeb (1998), the cultural norms in Saudi Islamic society encourage Saudis to conceal a 
considerable amount of their personal and familial information from the public domain. In particular, they are not comfortable with revealing full information regarding themselves or their families to outsiders, and they do not like intimate questions regarding their personal life and other personal queries because of their conservative nature (Culture Diversity, 2012). Nevertheless, a previous research revealed that Saudi university students disclosed about $60 \%$ of their basic identifiers, $77 \%$ of items categorised as sensitive personal information, and $50 \%$ of potentially stigmatising personal items on Facebook (Aljasir et al. 2017a). Such a finding may indicate that Saudi university students' social norms about disclosing personal information in this new online platform are shifting and diverging from the stereotypically reserved behaviour expected from them in their offline lives.

When asked about the reasons for these high levels of disclosure, the interviewees explained that they believed that sharing personal information is an essential requirement for being an active user. To benefit from Facebook, they indicated that they had to share personal information, such as their background information, activities, interests, and views. Such disclosure was perceived as a requirement to actively interact and engage in diverse activities on Facebook, such as establishing and maintaining relationships. Another factor that the participants indicated would motivate them to share and disclose more information was the encouragement they receive from other users through their likes and positive comments when information is disclosed. This finding is consistent with the main assumption of social penetration theory that individuals tend to disclose more personal information when they expect to receive encouragement (rewards) from others as an outcome of such disclosure (Altman \& Taylor, 1973). Thus, it seems that the more comments and likes users receive, the more likely they are to disclose information about themselves (Forest $\&$ Wood, 2012).

\subsection{Privacy Concerns on Facebook}

Although privacy has become a much-publicised topic in the field of new media studies, there is no consensus in the literature about its definition (Guo, 2010; Tarhini et al., 2016). Newell (1998) states that definitions given for privacy are so diverse and complex that it is impossible to evaluate them in a comprehensible way. However, there is some consensus that privacy relates to personal information, its control, and its disclosure (Tufekci, 2008). Li et al. (2014) define privacy concerns as an individual's general tendency to worry about the safety of his or her disclosed personal information. In terms of social penetration theory, privacy concerns could be evaluated from individuals' perceptions of the expected costs of using social media platforms.

When interviewing the current sample about their privacy concerns, all of the interviewees indicated that they are aware of the potential costs and negative consequences associated with disclosing their personal information. However, they differ in their privacy concerns. Some respondents indicated that they protected their privacy using the privacy settings on their accounts because they evaluated the potential negative consequences associated with disclosing their personal information as higher than their benefits from Facebook platform. Their uses of the settings ranged from hiding part of their contact information to blocking access to the entire account to non-friends. On the other hand, the rest of the respondents indicated that they are willing to jeopardise the privacy of their personal information to maintain the rewards they obtain as long as these rewards outweigh the expected costs from such disclosure. For instance, they indicated that sharing personal information, photos, and videos to keep in touch with friends and developing relationships with others outweighs the negative consequences associated with leaking personal information to undesired audiences. This phenomenon of increased public disclosure - termed 'radical transparency' - led Zuckerberg to argue that privacy is not a social norm anymore (Joinson, et al. 2011).

It should be noted that previous studies regarding the relationship between disclosure and privacy online have taken one of two stances. According to one viewpoint, although online users claim to be concerned about their online privacy, observing their actual online behaviour indicates that they disclose a considerable amount of information. Other studies have revealed that the level of disclosure online is negatively associated with privacy concerns. Thus, disclosing a high level of information online is associated with a low level of concern regarding privacy issues. The current findings contribute to this body of knowledge by revealing that although the majority of the current sample is aware of the potential costs and negative consequences associated with disclosing personal information, they are highly motivated to continue benefiting from Facebook by disclosing personal information in light of the costs and rewards equation.

\section{Discussing Social and Political Issues through Facebook}

According to Gil de Zúñiga, Jung, \& Valenzuela (2012), growing social media usage has led to a new debate regarding the overall benefits it brings to society by enabling individuals to be informed, discover shared causes, and contribute more to public life, versus its potential to increase the superficiality of relationships and distract users from focusing on and engaging in public affairs. The results of an earlier research revealed that Saudi university 
students use Facebook to satisfy their need to raise their concerns and discuss social and political issues, and a considerable portion of their status updates focus on such issues (Aljasir et al. 2017a). This section presents and discusses interviewees' perceptions of the freedom that Facebook offers them to discuss these issues and provides further insights into what they discuss on its platform for this purpose.

When interviewing respondents about the extent to which they freely discuss social and political issues, the students indicated that Facebook expands their opportunities to express their opinions freely regarding issues that were concerned about. However, they stressed that such freedom has its limit, for instance they should not cross the lines drawn by the state or their cultural and Islamic norms.

When interviewing the students about the political issues they discussed, they indicated that these related to global and local news updates. In global affairs, they indicated that they expressed their opinions on topics such as the election of the Islamic party in Egypt, their expectations for the Syrian revolutions, and the consequences of bringing down the regime in Yemen. They also engaged with other Facebook users, either in Saudi Arabia or other Arab countries, in discussing the reasons behind some events during the Arab Spring. Regarding local political issues, as the call for protests by Shiite minorities was the most significant political event in Saudi Arabia, the sample responses focused on this topic. For instance, Saudi university students mentioned the negative consequences in the Arab countries that went through the Arab Spring, such as Egypt, Yamen, Libya, and Syria, and how they suffered destruction in their countries.

Although the social issues raised covered a wide range, the respondents were not as critical of the state as those in other Arab countries have been. In neighbouring countries, social media has been used more directly to attack government officials and to blame the regimes for corruption (e.g. Alhammash 2012; Marzouki et al. 2012; Mansour 2012; Khamis, Gold, and Vaughn 2012). This finding is consistent with Boghardt's (2013) argument that the most popular Saudi social media content reveals interest in reforming policies, not in creating revolutionary change or promoting violent activism. The respondents' answers to the question regarding the issues raised, indicated that Saudi university students' demands concerned accelerating positive changes in the society.

\section{Conclusion}

A qualitative phenomenological approach was used to conduct the interviews. According to Wojnar \& Swanson (2007: 173), "phenomenology as a philosophical perspective has thrown light on previously ignored phenomena of human experience. ... At the core of phenomenology lies the attempt to describe and understand phenomena". Such an investigation typically involves collecting data using semi-structured interviews with the participants (Creswell, 2009). Therefore, one-to-one interviews were conducted in order to investigate in more detail some of the experiences the sample had had with Facebook. Twenty students (10 males and 10 females) indicated their willingness to participate.

In an earlier research, it was shown that Facebook is used to satisfy a wide range of gratifications, ranging from the creation and maintenance of friendships and family relations, education, romantic relationships, and the purchase of goods to discussing social and political issues (Aljasir et al., 2017a). A content analysis of status updates also revealed that 16 different status themes are posted ranging from simple greetings to the discussion of social and political issues (Aljasir et al., 2017b). The findings from this research shows how the adoption of the theoretical framework and methodological approach in this research can extend current knowledge and take an important step toward explaining what Saudi university students obtain from their usage of Facebook and its potential costs. The current research complements this picture by looking in more detail at Facebook usage and its compatibility with Saudi culture, the reasons behind Saudi university students' high levels of information disclosure, and their perceptions regarding the use of Facebook to discuss social and political issues.

The results reveal that Saudi university students have a positive attitude toward Facebook, regard it as an integral part of their daily lives, and do not consider deactivating or deleting their accounts. They appreciate the benefits of cross-cultural communication, which leads them to reject the idea of having a closed national social media platform. Because of its privacy settings and/or the increasing openness of Saudi society, Saudi university students indicated that Facebook does not contradict the values of their culture. The findings also reveal that Saudi university students were aware of some of the positive and negative consequences of using Facebook. The positive and negative cards chosen by the sample assisted in further investigating uses and gratification theory's assumptions on the usage of a social media platform.

When asked about the reasons for their high levels of disclosure and whether they hold privacy concerns regarding their disclosed information, the interviewees stated that such disclosure was necessary to be active users and 
maximise the rewards they obtain from Facebook. Despite acknowledging the potential costs and negative consequences of sharing personal information, the majority of the respondents did not conceal their personal information on their Facebook accounts, as long as the disclosure of such information did not come to cost them too much in comparison to what they were getting from its platform. Focusing on social and political issues, Saudi university students believe that Facebook enables them to to voice their opinions and concerns regarding social and political issues. The issues they discussed covered a wide range of topics.

Although this research set out to fill some gaps in knowledge regarding the reasons behind Facebook usage, it raised questions and highlighted new areas of interest that need further investigation: for example, this framework could be used to conduct research on other Saudi groups with different educational and age profiles. Conducting comparison studies across generations would add to the current findings and contribute to the media and communication research. It would also be interesting to carry out the same research with a similar sample or with young adults in a few years' time to see if there have been any cultural changes in their behaviour.

Since this research found that Facebook provided Saudi university students with opportunities that they could not obtain as easily in their offline worlds, such as discussion of social and political issues, it would be interesting to study the impact of the use of Facebook on Saudis' cultural behaviour online and whether such impact could be transferred to their offline lives. Thus, it would be interesting to investigate what is going to happen in the future as a result of the Saudi users' current experience with using Facebook. Future research could also look at investigating whether there is any evidence for radicalisation in young adults' behaviour based on their actions online.

The current results reveal that Saudi university students are willing to jeopardise the privacy of their personal information to maintain the rewards they obtain as long as these rewards outweigh the expected costs from such disclosure. As it seems that there is no endgame of privacy and the maintained equation of the rewards verses costs today may not be perceived as applicable in the coming future, it is worthwhile to investigate whether there will be a consensus among users on how to sustain a workable balance in favour of protecting the privacy of their personal information. As the Internet of Things arises and almost every aspect of individuals' daily lives will be based on them, it is also interesting to examine if these individuals will increasingly accept the exchange of their privacy for benefiting from such Internet services or whether they will have a strong desire to protect their personal information from 'dataveillance', a term referring to the surveillance of all the digital records of an individual's activities.

\section{References}

Al-Khateeb, S. (1998). Women, Family and the Discovery of Oil in Saudi Arabia. Marriage \& Family Review, 27(1-2), 167-189. https://doi.org/10.1300/J002v27n01_11

Al-Saggaf, Y. (2012). Social Media and Political Participation in Saudi Arabia: The Case of the 2009 Floods in Jeddah. In Proceedings Cultural Attitudes towards Communication and Technology Conference, held 18-20 June 2012 at Murdoch University, Perth, Australia.

Alhammash, M. (2012). The Arab Spring and Social Media. In Langmia, K., O’Brien, P., Sturgis, I. and Tyree, T. (Eds.), Proceedings of Second Annual Social Media Technology Conference \& Workshop. 'Social Media and Intersections between Politics, Culture and Law', held 27-28 September at Howard University, Washington, D.C, USA. Washington: Howard University 1, 3-10.

Aljasir, S., Bajnaid, A., Elyas, T., \& Alnawasrah, M. (2017a). Themes of Facebook Status Updates and Levels of Online Disclosure: The Case of University Students. International Journal of Business Administration, 8(7), 80. https://doi.org/10.5430/ijba.v8n7p80

Aljasir, S., Bajnaid, A., Elyas, T., \& Alnawasrah, M. (2017b). University Students Usage of Facebook: The Case of Obtained Gratifications and Typology of Its Users. Journal of Management and Strategy, 8(5), 30. https://doi.org/10.5430/jms.v8n5p30

Altman, I., \& Taylor, D. A. (1973). Social penetration: The development of interpersonal relationships. Holt, Rinehart \& Winston.

Ayres, L. (2008). Semi-structured Interview. in The SAGE Encyclopaedia of Qualitative Research Methods. ed. by Given, L. Thousand Oaks, CA: Sage Publications, 811-813

Barnes, S. B. (2006). A Privacy Paradox: Social Networking in the United States. First Monday, 11(9). https://doi.org/10.5210/fm.v11i9.1394

Barnum, C. M., \& Palmer, L. A. (2010, April). More than a feeling: understanding the desirability factor in user experience. In CHI'lO Extended Abstracts on Human Factors in Computing Systems (pp. 4703-4716). ACM. 
https://doi.org/10.1145/1753846.1754217

Benedek, J., \& Miner, T. (2002). Measuring Desirability: New methods for evaluating desirability in a usability lab setting. Proceedings of Usability Professionals Association, 2003(8-12), 57.

Chen, R., \& Sharma, S. K. (2013). Self-disclosure at social networking sites: An exploration through relational capitals. Information Systems Frontiers, 15(2), 269-278. https://doi.org/10.1007/s10796-011-9335-8

Chiang, H. S. (2013). Continuous Usage of Social Networking Sites: The Effect of Innovation and Gratification Attributes. Online Information Review, 37(6), 851-871. https://doi.org/10.1108/OIR-08-2012-0133

Chiou, W. B., \& Lee, C. C. (2013). Enactment of One-To-Many Communication May Induce Self-Focused Attention that Leads to Diminished Perspective Taking: The Case of Facebook. Judgment and Decision Making, 8(3), 372-380.

Cho, S. E. (2010). A cross-cultural comparison of Korean and* American social network sites: Exploring cultural differences in social relationships and self-presentation. Rutgers The State University of New Jersey-New Brunswick.

Christofides, E., Muise, A., \& Desmarais, S. (2009). Information Disclosure and Control on Facebook: Are They Two Sides of the Same Coin or Two Different Processes?. Cyber Psychology \& Behavior, 12(3), 341-345. https://doi.org/10.1089/cpb.2008.0226

Coleman, I. (2011). Saudi Arabia's Social Media Battles. Saudi Wave. Retrieved 13 April 2012, from http://www.saudiwave.com/Press/saudi-arabias-social-media-battles.html

Cozby, P. C. (1973). Self-disclosure: a literature review. Psychological bulletin, 79(2), 73. https://doi.org/10.1037/h0033950

Creswell, J. W., \& Inquiry, Q. (1998). Research Design: Choosing among five approaches.

Creswell, J.W. (2009). Research Design: Qualitative, Quantitative, and Mixed Methods ( ${ }^{\text {rd }}$ ed.). Thousand Oaks, CA: Sage Publication.

Culture Diversity. (2012). The Middle Eastern Community. Retrieved 19 August 2017, from http://www.culturediversity.org/mide.htm

Day, S. (2013). Self-disclosure on Facebook: How Much Do We Really Reveal?. Journal of Applied Computing and Information Technology, 17(1).

De Silva, T. (2010). Benefits of mixed methods in environmental reporting research. New Zealand: Lincoln University, 1-19.

DiCicco - Bloom, B., \& Crabtree, B. F. (2006). The qualitative research interview. Medical education, 40(4), 314-321. https://doi.org/10.1111/j.1365-2929.2006.02418.x

Doyle, W., \& Fraser, M. (2012). Facebook, Surveillance, and Power. In Wittkower, D. (Ed.), Facebook and Philosophy: What's on Your Mind? (pp.215-230). Illinois: Open Court Publishing.

El-Shenawi, E. (2014). Saudi Religious Police to Monitor Social Media?. Al-Arabiya, 22 February. Retrieved 12 September 2016, from http://english.alarabiya.net/en/media/digital/2014/02/22/Saudi-columnist-urges-religious-police-to-monitor-soci al-media.html

Ellison, N. B., Steinfield, C., \& Lampe, C. (2007). The Benefits of Facebook Friends: Social Capital and College Students' Use of Online Social Network Sites. Journal of Computer-Mediated Communication, 12(4), 1143-1168. https://doi.org/10.1111/j.1083-6101.2007.00367.x

Enli, G. S., \& Thumim, N. (2012). Socializing and Self-Representation Online: Exploring Facebook. Observatories, 6(1), 87-105.

Facebook. (2013). Data Use Policy. Retrieved 21 December 2016, from https://facebook.com/policy?page=all

Forest, A. L., \& Wood, J. V. (2012). When Social Networking Is Not Working Individuals with Low Self-Esteem Recognize but Do Not Reap the Benefits of Self-Disclosure on Facebook. Psychological Science, 23(3), 295-302. https://doi.org/10.1177/0956797611429709

Gauntlett, D. (2011). Making Is Connecting: The Social Meaning of Creativity from DIY and Knitting to YouTube and Web 2.0. London: Polity Press. 
Gil de Zúñiga, H., Jung, N., \& Valenzuela, S. (2012). Social Media Use for News and Individuals' Social Capital, Civic Engagement and Political Participation. Journal of Computer-Mediated Communication, 17(3), 319-336. https://doi.org/10.1111/j.1083-6101.2012.01574.X

Gillan, K., Pickerill, J., \& Webster, F. (2008). Anti-War Activism: New Media and Protest in the Information Age. New York, NY: Palgrave Macmillan. https://doi.org/10.1057/9780230596382

Govani, T., \& Pashley, H. (2005). Student Awareness of the Privacy Implications When Using Facebook. Unpublished Paper Presented at the "Privacy Poster Fair" at the Carnegie Mellon University School of Library and Information Science, 9. Retrieved 21 December 2016, from http://lorrie.cranor.org/courses/fa05/tubzhlp.pdf

Green, J., \& Thorogood, N. (2013). Qualitative methods for health research. Sage.

Gross, R., \& Acquisti, A. (2005, November). Information revelation and privacy in online social networks. In Proceedings of the 2005 ACM workshop on Privacy in the electronic society (pp. 71-80). ACM. https://doi.org/10.1145/1102199.1102214

Guillet, M. (2013). Penguins Instead of Protests. Doha Centre for Media Freedom. Retrieved 5 March 2016 , from http://www.dc4mf.org/en/content/penguins-instead-protests

Guo, Y. Y. (2010). The Privacy Issue on Social Network Sites: Facebook. Journal of Digital Research \& Publishing, 2 , 83-90.

Hammer, M. R., \& Gudykunst, W. B. (1987). The influence of ethnicity and sex on social penetration in close friendships. Journal of Black Studies, 17(4), 418-437. https://doi.org/10.1177/002193478701700403

Hays, R. B. (1985). A longitudinal study of friendship development. Journal of personality and social psychology, 48(4), 909. https://doi.org/10.1037/0022-3514.48.4.909

Higham, N. (2014). Web Brings more Freedom and more Surveillance'. BBC Vocativ, 1 April. Retrieved 20 April 2016, from http://www.bbc.com/news/magazine-26824802

Hofheinz, A. (2005). The Internet in the Arab World: Playground for Political Liberalization. International Politics and Society, 3(1), 78-96.

Iosifidis, P. (2011). The Public Sphere, Social Networks and Public Service Media. Information, Communication \& Society, 14(5), 619-637. https://doi.org/10.1080/1369118X.2010.514356

Jenkins, H. (2006). Convergence Culture: Where Old and New Media Collide. New York, NY: NYU Press.

Jin, S. A. A. (2013). Peeling back the multiple layers of Twitter's private disclosure onion: The roles of virtual identity discrepancy and personality traits in communication privacy management on Twitter. New Media \& Society, 15(6), 813-833. https://doi.org/10.1177/1461444812471814

Joinson, A. N., Houghton, D. J., Vasalou, A., \& Marder, B. L. (2011). Digital Crowding: Privacy, Self-Disclosure, and Technology. In Trepte, S., \& Reinecke, L. (Eds.), Privacy Online (pp. 33-45). New York, NY: Springer Berlin Heidelberg. https://doi.org/10.1007/978-3-642-21521-6_4

Jourard, S. M. (1964). The transparent self: Self-disclosure and well-being (No. 17). Van Nostrand.

Joyce, M. (2010). Digital Activism Decoded: The New Mechanics of Change. New York, NY: International Debate Education Association.

Karolak, M. (2013). Social Media and the Arab Spring: Searching for Emerging Identities in the Arab Gulf. St Antony's International Review, 9(1), 168-184.

Khamis, S., Gold, P. B., \& Vaughn, K. (2012). Beyond Egypt's "Facebook Revolution” and Syria's "YouTube Uprising:" Comparing Political Contexts, Actors and Communication Strategies. Arab Media and Society, 15, 1-30.

Kim, K. H., \& Yun, H. (2007). Cying for me, Cying for us: Relational dialectics in a Korean social network site. Journal of Computer-Mediated $\quad$ Communication, $\quad 13(1), \quad 298-318$. https://doi.org/10.1111/j.1083-6101.2007.00397.x

Kim, S. J., Bickart, B. A., Brunel, F. F., \& Pai, S. (2012). Can your business have 1 Million friends? Understanding and Using Blogs as one-to-one mass media.

Kisilevich, S., Ang, C. S., \& Last, M (2012). Large-scale Analysis of Self-Disclosure Patterns among Online Social Networks Users: A Russian Context. Knowledge and information systems, 32(3), 609-628. 
https://doi.org/10.1007/s10115-011-0443-z

Kolek, E. A., \& Saunders, D. (2008). Online Disclosure: An Empirical Examination of Undergraduate Facebook Profiles. NASPA Journal, 45(1), 1-25. https://doi.org/10.2202/0027-6014.1905

Krasnova, H., Günther, O., Spiekermann, S., \& Koroleva, K. (2009). Privacy Concerns and Identity in online Social Networks. Identity in the Information Society, 2(1), 39-63. https://doi.org/10.1007/s12394-009-0019-1

Labianca, G., \& Brass, D. J. (2006). Exploring the social ledger: Negative relationships and negative asymmetry in social networks in organizations. Academy of Management Review, 31(3), 596-614. https://doi.org/10.5465/AMR.2006.21318920

Limperos, A. M., Tamul, D. J., Woolley, J. K., Spinda, J. S., \& Sundar, S. S. (2014). "It's Not Who You Know, but Who You Add:" An investigation into the differential impact of friend adding and self-disclosure on interpersonal perceptions on Facebook. Computers in Human Behavior, 35, 496-505. https://doi.org/10.1016/j.chb.2014.02.037

Masi, A. (2013). Saudi Arabians Take to Twitter-8 Million Times - to Speak out Against Low Wages and a Rising Cost of Living. Vocativ, 8 August. Retrieved 2 April 2016, from http://www.vocativ.com/world/saudi-arabia-world/saudi-arabians-take-to-twitter-8-million-times-to-speak-out-a gainst-low-wages-and-a-rising-cost-of-living/

McEwan, B. (2011). Hybrid engagement: How Facebook helps and hinders students' social integration. In Higher Education Administration with Social Media (pp. 3-23). Emerald Group Publishing Limited.

Miladi, N. (2011). New media and the Arab Revolution: Citizen Reporters and Social Activism. Journal of Arab and Muslim Media Research, 4(2/3), 3-5.

Mohamed, A. (2011). Online Privacy Concerns among Social Networks' Users. Cross-Cultural Communication, 6(4), 74-89.

Morozov, E. (2009). Iran: Downside to the "Twitter Revolution". Dissent, 56(4), 10-14.

Morton, T. L. (1978). Intimacy and reciprocity of exchange: A comparison of spouses and strangers. Journal of Personality and Social Psychology, 36(1), 72.

Mustafa, S. E., \& Hamzah, A. (2011). Online Social Networking: A New Virtual Playground. International Proceedings of Economics Development \& Research, 5(2), 314-318.

Newell, P. B. (1998). A Cross-cultural Comparison of Privacy Definitions and Functions: A Systems Approach. Journal of Environmental Psychology, 18(4), 357-371. https://doi.org/10.1006/jevp.1998.0103

Nosko, A., Wood, E., \& Molema, S. (2010). All about Me: Disclosure in Online Social Networking Profiles: The Case of FACEBOOK. Computers in Human Behavior, 26(3), 406-418. https://doi.org/10.1016/j.chb.2009.11.012

Ntlatywa, P., Botha, R. A., \& Haskins, B. (2012). Claimed vs Observed Information Disclosure on Social Networking Sites. Information Security for South Africa, 1-6. https://doi.org/10.1109/ISSA.2012.6320443

Oct26driving (2013). Saudi Women Driving Campaign. Retrieved 12 December 2016, from http://linkis.com/www.oct26driving.com/pOy7

Olson, A. M. (2013). Facebook and social penetration theory (Doctoral dissertation, Gonzaga University).

Osman, F. Y., \& Abd Rahim, N. Z. (2012). Self-disclosure and Social Network Sites Users' Awareness. In the proceedings of the 2nd International Conference on Research and Innovation in Information Systems, held in 23-24 November at Kuala Lumpur, Malaysia.

Rathmell, A. (1997). Netwar in the Gulf. Jane's Intelligence Review, 29-32.

Reno, R. R., \& Kenny, D. A. (1992). Effects of self - consciousness and social anxiety on self - disclosure among unacquainted individuals: An application of the social relations model. Journal of Personality, 60(1), 79-94.

Romano, A. (2013). The Use of Social Media during a Crisis on Campus. Unpublished PhD Thesis. Muncie, IN: Ball State University.

Rubin, A. M. (1994). Media Uses and Effects: A Uses and Gratifications Perspective. In Bryant, J., \& Zillman, M. (Eds.), Media Effects: Advances in Theory and Research (pp. 417-436). Hillsdale, NJ: Erlbaum.

Samin, N. (2012). Saudi Arabia, Egypt, and the Social Media Moment. Arab Media and Society, 15. 
Saw, G., Abbott, W., Donaghey, J., \& McDonald, C. (2013). Social Media for International Students: It's not All about Facebook. Library Management, 34(3), 156-174. https://doi.org/10.1108/01435121311310860

Schanzer, J., \& Miller, S. (2012). Fatwa: Saudi Clerics, Wahhabi Islam and Social Media. New York, NY: FDD Press.

Scholz, T. (2012). Facebook as Playground and Factory. In Wittkower, D. (Ed.), Facebook and Philosophy: What's on Your Mind? Chicago, IL: Open Court Publishing.

Shi, P., Xu, H., \& Wang, N. (2013). Relationship Privacy in a Connected World: A Case of Facebook Friendship Page. A Poster at The Pennsylvania State University, University Park, PA, USA.

Shirky, C. (2011). The Political Power of Social Media. Foreign Affairs, 90, 28-41.

Silvermann, D. (1993). Interpreting qualitative data. Methods for analyzing Talk Text and Interaction.

Sorrell, J. M., \& Redmond, G. M. (1995). Interviews in qualitative nursing research: differing approaches for ethnographic and phenomenological studies. Journal of Advanced Nursing, 21(6), 1117-1122.

Starks, H., \& Brown Trinidad, S. (2007). Choose your method: A comparison of phenomenology, discourse analysis, and grounded theory. Qualitative health research, 17(10), 1372-1380.

Strater, K., \& Richter, H. (2007). Examining Privacy and Disclosure in a Social Networking Community. In Proceedings of the 3rd symposium on Usable Privacy and Security, held 18-20 July at Carnegie Mellon University in Pittsburgh, PA, USA. https://doi.org/10.1145/1280680.1280706

Strom, B. (2002). Communicator virtue and vice: Neglected constructs of relational communication?. Atlantic Journal of Communication, 10(1), 84-103.

Tang, J. H., \& Wang, C. C. (2012). Self-disclosure among bloggers: Re-examination of social penetration theory. Cyberpsychology, Behavior, and Social Networking, 15(5), 245-250.

Tesch, R. (1990). Qualitative analysis: Analysis types and software tools. Falmer Press, London. Thomas, SL, \& Zhang, L.(2005). Post-baccalaureate wage growth within four years of graduation: The effects of college quality and college major. Research in Higher Education, 46(4), 437-459.

Thibaut, J. W., \& Kelley, H. H. (1959). The social psychology of groups. 1959. New York: John Wiley.

Thotho, S. W. (2010). Information Disclosure on Facebook: A content Analysis of American and Kenyan user profiles. Unpublished dissertation. Muncie, IN: Ball State University

Tufekci, Z. (2008). Can You See Me Now? Audience and Disclosure Regulation in Online Social Network Sites. Bulletin of Science, Technology \& Society, 28(1), 20-36. https://doi.org/10.1177/0270467607311484

Tufekci, Z. (2012). Facebook, Youth and Privacy in Networked Publics. Month, 148, 36-37.

Wang, Z., Tchernev, J. M., \& Solloway, T. (2012). A Dynamic Longitudinal Examination of Social Media Use, Needs, and Gratifications among College Students. Computers in Human Behavior, 28(5), 1829-1839. https://doi.org/10.1016/j.chb.2012.05.001

Winter, S., Neubaum, G., Eimler, S. C., Gordon, V., Theil, J., Herrmann, J., ... Krämer, N. C. (2014). Another Brick in the Facebook Wall: How Personality Traits Relate to the Content of Status Updates. Computers in Human Behavior, 34, 194-202. https://doi.org/10.1016/j.chb.2014.01.048

Wojnar, D. M., \& Swanson, K. M. (2007). Phenomenology an Exploration. Journal of Holistic Nursing, 25(3), 172-180. https://doi.org/10.1177/0898010106295172

Wojnar, D. M., \& Swanson, K. M. (2007). Phenomenology: an exploration. Journal of holistic nursing, 25(3), $172-180$.

Yamani, M. (2010). Saudi Youth: The Illusion of Transnational Freedom. Contemporary Arab Affairs, 3(1), 7-20. https://doi.org/10.1080/17550910903454633

Young, A., \& Quan-Haase, A. (2009). Information Revelation and Internet Privacy Concerns on Social Network Sites: A Case Study of Facebook. In Proceedings of The Fourth International Conference on Communities and Technologies, held 25-27 June at Penn State University, Park, PA, USA. https://doi.org/10.1145/1556460.1556499

Zuckerman, E. (2014). Cute Cats to the Rescue? Participatory Media and Political Expression. In Allen, D. and Jennifer, L. (Eds.), Youth, New Media and Political Participation. Cambridge, MA: MIT Press. 